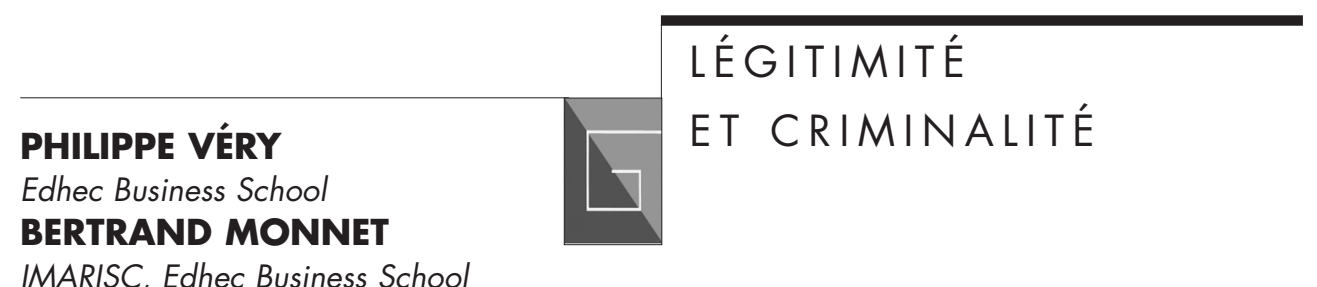

\title{
Quand les organisations rencontrent le crime organisé
}

Les organisations doivent-elles aujourd'hui craindre les groupes criminels organisés? À partir d'une méthodologie combinant des études de cas et une revue de presse, les auteurs proposent un modèle décrivant les différents types de crimes perpétrés contre les organisations et leurs impacts. Cette recherche montre qu'il n'existe plus deux mondes parallèles, le légal et l'illégal, mais un monde des affaires de plus en plus pénétré par le crime organisé. 
$\mathrm{E}$ ntreprise et crime organisé: deux mondes qui s'ignorent? Dépasser ce cliché rassurant est urgent: l'entreprise est la proie constante de prédateurs criminels de tous types qui chaque jour menacent un peu plus le fonctionnement de l'économie internationale. En témoignent quatre constats simples de l'emprise criminelle sur des enjeux fondamentaux de l'entreprise.

- À l'heure du baril de brut à plus de 50 dollars, les plus grandes compagnies pétrolières opèrent massivement en territoire criminalisé: terrorisme islamiste en Arabie Saoudite, piraterie en Indonésie, kidnapping en Colombie, vol massif au Nigeria (sixième exportateur de l'Opep où plus de $4 \%$ de la production réalisée par les majors a été piratée en 2004 !),...

- Deux déterminants-clés de la mondialisation sont, par ailleurs, gravement menacés par le crime organisé. Le développement du marché global s'accompagne à l'évidence de celui de la contrefaçon, spécifiquement en Inde et en Chine, cibles prioritaires des plus grandes entreprises occidentales. L'indispensable transfert de compétences vers de nouvelles zones d'opération que réalisent concrètement expatriés et voyageurs d'affaires étant, quant à lui, rendu dangereux donc coûteux par une nouvelle composante du risque/pays: Amérique du $\mathrm{Sud} /$ risques enlèvement contre rançon, pays musulmans/risques d'attentats, Afrique de l'Ouest/risques d'insurrection généralisé.

- L'énorme potentiel de marché créé par le processus d'élargissement de l'Union européenne va immanquablement exposer les plus grandes entreprises occidentales à des acteurs criminels fortement ancrés dans le secteur privé et la sphère politique des nou- veaux et futurs entrants, du crime organisé roumain à la mafia albanaise.

- La taille des acteurs criminels ne cesse d'augmenter et certains réalisent un chiffre d'affaires - estimé - supérieur au PIB de certains États. Par exemple, le chiffre d'affaires de la N'Dranghetta, mafia calabraise, est estimé à 35 milliards alors que le PIB de la Calabre est de 29 milliards d'euros! Ce sont donc de très riches organisations, souvent opérant à l'international, qui s'attaquent à l'entreprise.

Ces quelques arguments montrent que la maîtrise des risques criminels pesant sur les organisations constitue un enjeu majeur du management international. Or, force est de constater que la recherche académique en management s'est très rarement intéressée à cette question. L'objectif de cet article consiste à explorer ce champ méconnu, de manière à le structurer et à élaborer de futurs programmes de recherche. Pour cela, nous avons mené des investigations selon deux axes:

- proposer une typologie des crimes perpétrés contre les organisations;

- analyser les impacts de chaque type de crime sur l'organisation.

Ces investigations se sont appuyées sur une méthodologie double, combinant revue de presse et études de cas effectuées sur le terrain. Mais au préalable, nous avons dressé l'état des connaissances acquises sur le sujet.

\section{Crime et groupe criminel organisé}

La recherche est centrée sur les crimes touchant une cible particulière, l'organisation, et commis par un groupe particulier d'individus, le groupe criminel organisé.

Par crime nous entendons ici toute action illégale qui est perpétrée contre l'organisa- 
tion: vol de marchandises, enlèvement d'employé... L'auteur du crime est donc punissable par la justice puisqu'il enfreint la loi.

Par groupe criminel organisé, nous entendons les groupes correspondant à la définition élaborée par la Convention des Nations unies :

«L'expression "groupe criminel organisé" désigne un groupe structuré de 3 personnes ou plus existant depuis un certain temps et agissant de concert dans le but de commettre une ou plusieurs infractions graves ou infractions établies conformément à la présente convention, pour en tirer, directement ou indirectement, un avantage financier ou un autre avantage matériel.» (Convention des Nations unies contre la criminalité transnationale organisée, Palerme, décembre 2000, Article 2: terminologie).

Nous avons ensuite utilisé le cycle d'ascension criminelle décrit par Raufer et Quéré (2000) afin de cerner avec davantage de précision les acteurs inclus dans l'étude. Raufer et Quéré (2000) considèrent sept paliers d'évolutions possibles.

Étape 1: sur le seuil d'une « carrière » criminelle; l'individu est impliqué dans le proxénétisme, les vols, la revente de drogue, etc. L'activité est impulsive et discontinue. Il appartient éventuellement à un groupe peu structuré ou temporaire.

Étape 2 : crimes supposant structure et capital; l'individu développe une activité organisée et durable: vols à main armée planifiés, trafic de stupéfiants ou de voitures volées, etc. il possède des capacités de commandement, gère un territoire et structure son organisation en utilisant des complicités dans la société civile
Étape 3 : besoins de rentes complémentaires stables; l'individu n'est plus un amateur, il est lucide sur la nature criminelle de ses actions, il a besoin d'argent pour jouer « les grands seigneurs » et rétribuer hommes de mains et complices. Il s'assure un flux constant d'argent liquide en développant des activités comme l'extorsion ou les machines à sous. Son organisation est bien huilée.

Étape 4: S'infiltrer dans l'économie légitime; l'individu s'achète une façade légale (commerce, entreprise) qui facilite le recyclage de l'argent criminel et l'apprentissage de nouveaux moyens d'enrichissement.

Étape 5: activités prédatrices visant la société et l'État; l'individu se lance dans les fausses factures, les escroqueries à la Sécurité Sociale, le détournement de subventions; C'est le summum de la réussite criminelle dans de nombreux pays.

Étape 6: les cartels et sociétés criminelles analogues; cette étape n'est possible que dans certains pays: Colombie, Russie, Mexique. L'individu gère un empire d'apparence légale par prête-noms interposés, avec des ramifications internationales. Il tisse des liens avec les mondes politiques et économiques. Il continue à corrompre, intimider et trouve de nouveaux débouchés comme la prédation de marchés publics. C'est le summum de la réussite dans les pays cités.

Étape 7 : le Gotha criminel, les mafias; cette étape n'est pas atteignable pour la plupart des individus. Elle requiert d'être né au sein de la « famille ». Organisation cloisonnée, rituelle, au pouvoir important, ancrée dans l'histoire d'un pays, elle survit ou revit quand sa tête est coupée, contrairement aux 
autres organisations criminelles. C'est l'aristocratie du crime organisé (Raufer, 2005, p. 19).

Dans le cadre de notre recherche, nous avons retenu les groupes d'acteurs ayant atteint au minimum l'étape 3 du cycle, « besoins de rentes complémentaires stables », stade auquel le groupe criminel a développé une organisation structurée et agit criminellement de façon consciente. Notre population d'acteurs criminels comprend donc:

- les mafias ;

- les gangs, tels les gangs de motards impliqués dans les trafics de drogue ;

- des tribus et communautés locales, organisées de façon délibérée pour commettre des délits récurrents ;

- les bandes armées dégénérées: il s'agit des armées « révolutionnaires » qui ont abandonné leur objectif politique initial pour un objectif économique, comme les rebelles du NPDVF au Nigéria ;

- des réseaux terroristes organisés pour agir de façon récurrente.

Notons que plus on monte dans la hiérarchie du crime, plus les acteurs ont en général développé une façade respectable. Les Yakusas, mafia Japonaise, possèdent ainsi des entreprises « légales » dans l'immobilier ou la construction (Pierrat et Sargos, 2005). La secte Moon mêle de façon floue des activités criminelles et une présence forte dans la chaîne d'approvisionnement du sushi aux États-Unis (Dacin et al., 2007). Le contrôle d'entreprises « légales» permet, soit de masquer des trafics illicites, soit de se présenter de façon respectable et de fournir un « alibi » aux sources d'enri- chissement personnel des leaders du groupe criminel.

Sont exclus du champ de notre analyse:

- les individus isolés, comme un voleur ou un hacker, qui s'en prennent à l'entreprise ; - les employés commettant des crimes de leur propre chef; toutefois, un employé est parfois contraint par une organisation criminelle (chantage, pression sur la famille, etc.) ou corrompu pour agir au détriment de son entreprise. Si l'opération est pilotée par une communauté criminelle organisée, elle est incluse dans notre champ de recherche ;

- les entreprises « honnêtes $»^{1}$ accusées de crime, comme les accusations mutuelles de " concurrence déloyale », pour cause de financement sur fonds étatiques, proférées par Airbus et Boeing. Ces cas sont rejetés même si l'une des entreprises est condamnée pour pratique frauduleuse. Nous ne considérerons que les crimes où l'intervention d'un « groupe criminel organisé » est avérée.

Dans la littérature en gestion, le champ de recherche sur le crime commis envers l'organisation peut être scindé en trois catégories :

- les pratiques frauduleuses internes aux entreprises; les affaires Enron, Worldcom ou Tyco ont donné naissance à des recherches sur les pratiques de gouvernance (Morrison, 2004; Deakin et al., 2004; Kaufman et al., 2005). D'autres travaux ont été menés sur le rôle des whistle blowers (Gundlash et al., 2003; Vershoor, 2003), ces dénonciateurs que Claveau et Séville appellent « les tireurs de sonnette d'alarme » (2004) ;

1. Plus précisément, nous considérons une entreprise comme « honnête » lorsqu'elle n'a pas de lien actionnarial prouvé avec un groupe criminel organisé. 
- la corruption des États; le criminel ${ }^{2}$ est généralement un fonctionnaire d'État qui s'enrichit pour so compte ou celui de son gouvernement (Schleifer et Vishny, 1993). La plupart des recherches traite du lien entre le niveau de corruption dans un pays et les flux étrangers d'investissements vers ce pays; les quelques recherches s'intéressant à l'organisation examinent les modes d'entrée appropriés en fonction du niveau de corruption dans le pays d'accueil (Doh et al., 2003; Uhlenbruck et al., 2006) ;

- depuis les attentats du 11 septembre 2001, le risque terroriste et le cyber-crime. Un numéro spécial de Journal of International Management (vol. 11, $\mathrm{n}^{\circ} 4,2005$ ) a rassemblé les premières recherches dans ce domaine. Seule cette dernière catégorie de crime correspond à notre problématique. Étudions-là plus avant.

\section{Recherches en gestion}

Dans le numéro spécial de Journal of International Management (2005), plusieurs articles théoriques contiennent des éléments de réflexion utiles pour notre recherche. Czinkota et al. (2005) ont étudié les impacts du terrorisme sur la stratégie marketing et la production. Leur construction théorique les mène à supposer que la saillance du risque terroriste influence toutes les composantes de la chaîne de valeur qu'ils ont étudié. L'intérêt de leur recherche pour notre propre travail réside dans la distinction effectuée entre impacts directs et indirects. Par impact direct les auteurs entendent les conséquences immédiates de l'attentat terrorisme (destruc- tion du siège, décès des employés, etc.). Limpact indirect est conçu comme les conséquences qui s'enchaînent derrière l'impact direct. Dans leur étude, les auteurs évoquent la chute de la consommation aux États-Unis suite aux attentats du 11 septembre. Traitant des conséquences liées à ce même évènement, Spich et Grosse (2005) évoquent deux types de surcoûts pour les entreprises américaines:

- un surcoût micro-économique, dû aux mesures mises en œuvre par l'entreprise pour protéger des hommes et ses sites;

- un surcoût macro-économique, causé par la mise en place de la politique américaine de sécurité qui requiert de s'adapter à de nouvelles réglementations.

Faisant un parallèle entre coûts et impacts, notre recherche est centrée sur les impacts micro-économiques, directs ou indirects, que l'action criminelle exerce sur l'entreprise. Nous ne traitons pas les coûts qui affectent l'ensemble de la population des entreprises d'un pays.

Enfin, Spich et Grosse (2005) évoquent une influence de la politique américaine de sécurité sur le chiffre d'affaires des entreprises nationales, qui peut décroître (clients ne voulant plus acheter américain) ou croître (opportunités de nouveaux produits ou services de sécurité).

\section{Recherches en criminologie}

$\mathrm{Si}$ les recherches en gestion nous aident à identifier les impacts possibles de l'action criminelle, elles se révèlent peu utiles pour dresser un panorama des crimes commis

2. La corruption est perçue comme un crime par la plupart des multinationales occidentales, alors qu'elle peut très bien être perçue comme une coutume locale par les citoyens du pays. D'où les difficultés à définir le crime. Un crime se définit par rapport à une législation donnée, celle d'un pays dans notre cas. Le lecteur se réfèrera à la discussion des résultats pour de plus amples développements sur ce point. 
contre les organisations par les groupes criminels organisés. C'est pourquoi nous nous sommes plongés dans les travaux en criminologie. Les criminologues s'intéressent à l'identification des groupes criminels, à la compréhension de leurs motivations et à un décryptage de leurs modalités d'action. Cette littérature ne traite pas spécifiquement le problème de l'action criminelle dirigée contre l'organisation, mais elle fournit des pistes de réflexions grâce au décryptage des modes d'action. C'est pourquoi, avant, pendant et après l'étude empirique, nous l'avons consultée pour préparer nos enquêtes et étayer nos conclusions ${ }^{3}$.
En résumé, deux champs de littérature distincts ont été utilisés pour répondre à nos deux questions de recherche:

- les travaux des criminologues constituent une base solide de réflexion pour dresser une typologie de l'action des groupes criminels organisés contre les organisations; - les travaux en gestion sont utiles pour identifier les impacts possibles de cette action criminelle.

À partir de ces recherches, nous avons établi un plan d'investigation empirique (voir encadré « Méthodologie ») qui nous a permis d'aboutir aux résultats décrits cidessous.

\section{MÉTHODOLOGIE}

Pour explorer ce champ inconnu, nous avons combiné une revue de presse et l'examen de cas réels.

La revue de presse a consisté à collecter tous les articles publiés dans le Wall Street Journal entre le 11 septembre 2001 et le 20 février 2007 qui décrivaient un crime correspondant à notre objet de recherche. Seuls les articles bien documentés et non redondants furent retenus, soit 53 articles décrivant 64 crimes différents. C'est en agrégeant ces 64 crimes en 7 catégories que nous avons élaboré la typologie des crimes commis contre les organisations par les groupes criminels organisés. Cette typologie fut ensuite confrontée à la littérature en criminologie pour asseoir sa pertinence. L'étude de cas réels a consisté à analyser 10 crimes, aussi divers que possible, commis par une variété de groupes contre diverses organisations et dans différents endroits de la planète. Ces 10 cas couvraient toutes les catégories de la typologie des crimes. Nous avons suivi la méthodologie d'Eisenhardt (1989) pour mener à bien ces investigations et analyses. Nous avons parfois emprunté à la criminologie ses méthodes pour analyser une scène de crime et identifier des preuves de l'acte délictueux. En effet, dans certains cas, les entreprises ne comprenaient pas vraiment ce qui leur était arrivé. Les témoins ont été interrogés, y compris les responsables de certaines organisations criminelles lorsque c'était possible. L'un des auteurs de cette recherche est expert en criminologie; c'est donc lui qui a conduit les enquêtes avec le concours des organisations étant l'objet d'action criminelle. Pour chaque crime étudié, nous avons tenté de repérer les impacts sur l'organisation ciblée.

3. Nous avons choisi de ne pas citer les très nombreux écrits consultés. En effet, la littérature pertinente dépend du cas traité : quelle est la nature du crime et qui en sont les auteurs? Pour chaque événement étudié, nous avons donc recherché des informations différentes, en recoupant connaissance du pays, connaissance de l'organisation criminelle et connaissance du mode d'action employé. 
Tableau 1 - Typologie des actions criminelles sur l'organisation

\begin{tabular}{|c|c|c|c|c|c|c|}
\hline Type & Intitulé & Pratiques communes & $\begin{array}{l}\text { Localisations } \\
\text { principales }\end{array}$ & $\begin{array}{c}\text { Organisations } \\
\text { criminelles impliquées }\end{array}$ & Objectif du crime & $\begin{array}{c}\text { Exemple cité dans } \\
\text { l'article }\end{array}$ \\
\hline 1 & $\begin{array}{l}\text { Chasse au } \\
\text { trésor }\end{array}$ & $\begin{array}{l}\text { Extorsion simple, vente } \\
\text { forcée, travail forcé }\end{array}$ & $\begin{array}{l}\text { Extorsion simple ou vente forcée: } \\
\text { surtout pays développés (USA, } \\
\text { Italie, Japon, etc.) } \\
\text { Travail forcé: Myanmar, Afrique } \\
\text { Noire, Nouvelle Guinée, etc. }\end{array}$ & $\begin{array}{l}\text { Très diverses: mafias, } \\
\text { gangs, communautés, } \\
\text { bandes organisées }\end{array}$ & $\begin{array}{l}\text { S'enrichir en rackettant les } \\
\text { entreprises présentes sur le } \\
\text { territoire de l'acteur criminel }\end{array}$ & $\begin{array}{l}\text { Pétrole au Nigéria: } \\
\text { les travailleurs } \\
\text { fantômes du delta } \\
\text { du Niger }\end{array}$ \\
\hline 2 & $\begin{array}{l}\text { Chasse au } \\
\text { produit }\end{array}$ & $\begin{array}{l}\text { Vols de produits et } \\
\text { composants, bunkering, } \\
\text { piraterie maritime, } \\
\text { routière }\end{array}$ & $\begin{array}{l}\text { Vols: partout } \\
\text { Bunkering: sites pétrolifères } \\
\text { Piraterie maritime: Mallaca, Mer } \\
\text { de Chine, Golfe du Yemen, etc. } \\
\text { Piraterie routière: surtout pays } \\
\text { développés }\end{array}$ & $\begin{array}{l}\text { Très diverses: mafias, } \\
\text { gangs, bandes } \\
\text { organisées }\end{array}$ & $\begin{array}{l}\text { S'enrichir par la revente des } \\
\text { produits dérobés }\end{array}$ & $\begin{array}{l}\text { Bunkering au } \\
\text { Nigeria }\end{array}$ \\
\hline 3 & $\begin{array}{l}\text { Chasse au } \\
\text { marché }\end{array}$ & $\begin{array}{l}\text { Piratage de marchés } \\
\text { publics et de } \\
\text { privatisations }\end{array}$ & $\begin{array}{l}\text { Marchés publics: pays } \\
\text { développés } \\
\text { Privatisations: pays en sortie de } \\
\text { guerre ou entrant dans l'économie } \\
\text { de marché }\end{array}$ & $\begin{array}{l}\text { Mafias, groupes } \\
\text { criminels locaux }\end{array}$ & $\begin{array}{l}\text { S'enrichir par appropriation } \\
\text { du marché }\end{array}$ & $\begin{array}{l}\text { Privatisation dans } \\
\text { les Balkans }\end{array}$ \\
\hline 4 & $\begin{array}{l}\text { Chasse à } \\
\text { l'homme }\end{array}$ & Kidnapping et rançon & $\begin{array}{l}\text { Amérique du Sud, Russie, } \\
\text { Afrique noire, Pakistan, } \\
\text { Afghanistan }\end{array}$ & $\begin{array}{l}\text { Très diverses: mafias, } \\
\text { gangs, bandes } \\
\text { organisées }\end{array}$ & $\begin{array}{l}\text { S'enrichir par le biais d'une } \\
\text { rançon exigée contre } \\
\text { libération de l'otage }\end{array}$ & $\begin{array}{l}\text { Kidnapping en } \\
\text { Colombie }\end{array}$ \\
\hline 5 & $\begin{array}{l}\text { Chasse aux } \\
\text { savoir-faire }\end{array}$ & $\begin{array}{l}\text { Espionnage industriel et } \\
\text { contrefaçon }\end{array}$ & Partout & $\begin{array}{l}\text { Mafias, groupes } \\
\text { criminels puissants }\end{array}$ & $\begin{array}{l}\text { S'enrichir en revendant un } \\
\text { savoir-faire ou en } \\
\text { concurrençant l'entreprise } \\
\text { grâce à ce savoir-faire }\end{array}$ & $\begin{array}{l}\text { Contrefaçon de } \\
\text { cigarettes chez les } \\
\text { futurs entrants } \\
\text { européens }\end{array}$ \\
\hline 6 & $\begin{array}{l}\text { Chasse au } \\
\text { symbole }\end{array}$ & $\begin{array}{l}\text { Attentat contre des } \\
\text { employés ou des sites } \\
\text { d'entreprises }\end{array}$ & Partout & Groupes terroristes & $\begin{array}{l}\text { Détruire pour raison } \\
\text { idéologique }\end{array}$ & $\begin{array}{l}\text { Tentatives } \\
\text { d'attentat contre le } \\
\text { rallye Paris-Dakar }\end{array}$ \\
\hline 7 & Parasitisme & $\begin{array}{l}\text { Trafics de drogue, armes, } \\
\text { êtres humains,... ou } \\
\text { blanchiment d'argent sale }\end{array}$ & Partout & $\begin{array}{l}\text { Mafias, groupes } \\
\text { criminels impliqués } \\
\text { dans ces trafics }\end{array}$ & $\begin{array}{l}\text { Utiliser les « tuyaux » de } \\
\text { l'entreprise pour faciliter le } \\
\text { déploiement d'activités } \\
\text { criminelles lucratives }\end{array}$ & $\begin{array}{l}\text { Trafic de drogue } \\
\text { chez un producteur } \\
\text { de métaux }\end{array}$ \\
\hline
\end{tabular}




\section{PÉTROLE AU NIGERIA: LES TRAVAILLEURS FANTÔMES DU DELTA DU NIGER}

Avec une production de pétrole brut de 2,6 millions de barils/jour, le Nigeria est le sixième exportateur de l'Opep. Tout est - théoriquement - fait pour que cette manne profite aux habitants des 36 États fédérés du pays, et notamment à ceux peuplant le delta du Niger d'où est extraite la grande majorité du brut. Une loi garantit, en outre, le reversement de $13 \%$ des royalties versées par les compagnies pétrolières aux gouvernements des 36 États fédérés du pays. Cet énorme potentiel de développement est totalement ruiné par une corruption record qui touche l'État fédéral mais aussi et surtout les gouvernements locaux. Et des $13 \%$ des royalties censés permettre de sortir de la misère les populations du delta, et spécifiquement les communautés ethniques Ijaw, ne reste que moins de $1 \%$. Si une partie de la différence ne quitte pas les caisses de l'État central, toujours décidé à maintenir sous dépendance une région historiquement sécessionniste et agitée, l'essentiel de l'argent détourné part dans les poches des gouverneurs eux-mêmes.

Pour ne pas mourir lentement, les populations du delta du Niger ont deux choix. Le premier consiste à résister à l'État kleptocrate et aux gouverneurs corrompus en brisant par la force le système de vol organisé de l'argent public. Comme cela sera décrit plus loin, la nature elle-même criminelle des mouvements rebelles censés défendre cette noble cause annule cette possibilité. Sans espoir d'établir une juste redistribution, reste la possibilité du prélèvement à la source: les compagnies pétrolières et leurs sous-traitants parapétroliers.

Les communautés ethniques du delta pratiquent une forme locale et atténuée d'extorsion, généralement payé en riz ou en essence. Mais la principale technique qu'elles utilisent pour vivre des compagnies pétrolières est de recourir de façon massive et organisée à l'emploi forcé.

Des négociations sont ainsi régulièrement menées entre le roi de chaque communauté et les compagnies pétrolières et parapétrolières opérant sur le territoire de celle-ci, on shore ou off shore. Celles-ci visent à fixer le nombre d'employés devant impérativement être recrutés au sein de la communauté pour les différents chantiers d'exploration ou de production conduits par les compagnies. Ce type de recrutement peut se concevoir pour certains emplois non qualifiés, mais devient problématique pour les postes d'ouvriers spécialisés, de techniciens ou d'employés administratifs. Ce d'autant plus que les recrutements exigés dépassent bien souvent le nombre d'employés prévu pour le chantier... Une seule solution pour les compagnies : effectuer ces recrutements en priant la majorité des heureux élus de bien vouloir rester chez elle, et bien sûr payer parallèlement une main-d'œuvre qualifiée à chaque poste.

Un nombre précis de ces travailleurs fantômes est prévu pour chacun des groupes sociaux composant la communauté. Tout refus de procéder aux recrutements exigés entraîne à court ou moyen terme une réaction violente. Celle-ci peur revêtir plusieurs formes. La grève sauvage est le moyen le plus couramment utilisé. Une autre réaction fréquente des communautés à la résistance au recrutement forcé est l'envahissement des sites de productions: champs pétroliers onshore ou offshore, stations de pompage, plateformes, barges, bateaux de liaison, compounds, etc. La violence est alors systématiquement employée, incendie et pillage intégral transformant le site visé en paysage lunaire. Malgré leur forte expérience du terrain, les cadres de Total se souviennent encore de l'attaque du champ OML 56, dévasté en 2002 
par des centaines de jeunes hommes (les youths) de la communauté Itsekeri avoisinante. Quatre ans après les faits, et malgré l'intérêt stratégique de ce champ pour Total ${ }^{4}$, la compagnie ne se risque toujours pas à y retourner.

Selon les déclarations du directeur général d'Elf Petroleum Nigeria Limited (EPNL, la filiale nigériane de Total), cette prédation exercée par les communautés double a minima les coûts de production de la compagnie dans le pays.

\section{Typologie des actions criminelles}

Rappelons que l'objectif de cette typologie consiste à mettre de l'ordre dans les attaques subies par les entreprises de la part des organisations criminelles. L'entreprise est source de richesses et symbole du capitalisme. Quelles sont ces richesses et symboles convoités - ou abhorrés - par les organisations criminelles?

Nous avons au final identifié 7 types d'action criminelle qui sont présentés selon la richesse ou le symbole visé: chasse au trésor, chasse au produit, chasse au marché, chasse à l'homme, chasse à l'image et parasitisme. Le tableau 1 présente cette typologie et une description sommaire des acteurs impliqués dans chaque type de crime.

Ces 7 types de crime sont explicités ciaprès et illustrés par un cas étudié.

\section{Type 1 : la chasse au trésor}

La chasse au trésor consiste à ponctionner directement les ressources financières de l'entreprise. Dans cette catégorie, se retrouvent les extorsions simples comme le pizzo italien et le racket par intrusion dans l'entreprise qui prend deux formes:

- vente forcée de prestations de services: sécurité, équipements de bureau, - emploi forcé: les travailleurs fantômes.
La prédation efficace des ressources financières impose que l'entreprise ciblée donne la somme exigée. Ce don n'est bien sûr pas effectué de plein gré mais sous la contrainte du prédateur. Ce mécanisme est celui de l'extorsion (ou racket) : application sur l'entreprise d'une contrainte suffisamment forte (torsion) pour qu'elle affecte d'ellemême une partie de ses revenus à des opérations sortant (ex) de son fonctionnement logique.

\section{Type 2 : la chasse au produit}

La chasse au produit regroupe toute action visant à s'approprier illégalement les produits, les composants et matières premières entrant dans leur fabrication. Ce sont les inputs et outputs qui sont donc visés. L'intérêt réside dans la valeur marchande de ces ressources. Dans cette catégorie, on trouve toutes formes de vol, allant jusqu'à la piraterie maritime ou terrestre.

\section{Type 3 : la chasse au marché}

La chasse au marché consiste à s'approprier les marchés visés par l'entreprise, en général par l'intermédiaire de sociétés à la façade honorable et respectable. Deux types d'action entrent dans cette catégorie:

- le piratage de marchés publics,

- le piratage de privatisations.

4. Total ne possède que deux concessions onshore au Nigeria: OML 56 et OML 58, la compagnie étant, par ailleurs, concessionnaire d'importants champs offshore. 


\section{BUNKERING AU NIGERIA}

Bunkering désigne l'action de vol de pétrole brut par destruction partielle ou piratage de pipe-line. Selon des sources concordantes 5 , le volume de brut volé par bunkering dans le delta du Niger se situe dans une fourchette de 5 à $10 \%$ de la production totale réalisée au Nigeria, soit entre 130000 et 260000 barils/jour. À 60 dollars/baril, le chiffre d'affaires théorique du bunkering représente de 7,8 à 15,6 millions dollars/j, soit 2.8 à 5.6 milliards dollars/an.

S'il génère des bénéfices colossaux pour les différents acteurs qui le pratiquent, le bunkering entraîne un manque à gagner de même proportion pour les compagnies pétrolières volées. Chaque année, certaines des plus grandes entreprises du monde comme Exxon, Shell, Eni, Chevron ou Total perdent ainsi entre 5 et $10 \%$ de leur chiffre d'affaires théorique « avant bunkering » sur l'une de leurs zones de productions stratégiques.

Si différentes techniques de bunkering existent, la principale d'entre elles consiste à pirater des oléoducs de gros volume en soudant une dérivation clandestine sur une de leurs vannes. Une fois le brut piraté, il est mis en baril à proximité du pipeline, et chargé sur des barges de tonnage moyen $(1000 \mathrm{t})$. À la sortie du delta du fleuve, le brut est transbordé ou pompé des barges vers des pétroliers de faible tonnage (5000 t) qui l'acheminent vers ses raffineries de destination (régionales, mais aussi européennes).

Les opérations décrites plus haut sont longues et bruyantes. Elles mobilisent des dizaines d'acteurs, des moyens logistiques majeurs, du groupe électrogène au petit tanker, et nécessitent la réalisation d'opérations techniques complexes, de la soudure sur pipe line au pompage de brut afin de transbordement. Elles nécessitent enfin la délivrance frauduleuse de vrais certificats d'exportation, afin que le pétrole volé puisse être vendu sans attirer l'attention d'acheteurs tenus au respect de normes de traçabilité du brut qu'ils raffinent.

Bien loin de n'impliquer que des trafiquants locaux ou des groupes rebelles du delta, le bunkering est donc une activité criminelle organisée. De telles opérations nécessitent à l'évidence de fortes complicités, chez militaires et politiques.

\section{Type 4: la chasse à l'homme}

La chasse à l'homme est une forme particulière d'extorsion appelée couramment « kidnapping et rançon ${ }^{6}$. Ici les employés représentent un moyen d'extorquer des fonds en jouant sur la vie des otages et la responsabilité de l'entreprise vis-à-vis de ses troupes. La richesse de 1'entreprise, ce sont ses hommes qui représentent une monnaie d'échange. Nous avons décidé d'en faire un type à part car les conséquences pour l'entreprise sont différentes de celles rencontrées lors d'extorsion du type chasse au trésor.

5. Dirigeants de filiales de compagnies pétrolières et ex-bunkerers. 


\section{PIRATAGE DE PRIVATISATION DANS LES BALKANS}

Un grand groupe européen - que nous nommerons WB - tenta en 2006 de pénétrer dans l'un des pays balkaniques en rachetant la plus grosse société locale dans son industrie. Cette société appartenait à l'État qui avait décidé de la vendre, dans le cadre d'une politique de privatisation. Le groupe WB fit toutes les analyses classiques préalables à une acquisition, à partir des informations dont il pouvait disposer. Il évalua le prix à payer pour la cible et prit les contacts locaux nécessaires afin de signaler son intérêt pour la cible. C'est alors que la rumeur se mit à courir: la société à vendre avait été l'objet de pratiques comptables frauduleuses en interne et était au bord de la faillite. Les journaux locaux firent une campagne de presse en ce sens. Devant la levée de boucliers à l'encontre de l'entreprise, l'État demanda un audit, confirma la situation de faillite et bien évidemment retira cette compagnie de la liste des privatisations. Elle fut attribuée judiciairement, pour une somme modique, à un groupe local.

L'enquête commanditée par WB prouva que toute cette opération était un coup monté. La cible était en bonne santé. Il y avait collusion entre le gouvernement corrompu, des experts juridiques et les principaux médias du pays pour orchestrer ce subterfuge. Mais l'affaire n'était pas un simple cas de corruption. Il fut démontré que le repreneur était un groupe tenu par une bande criminelle puissante localement, ayant des liens étroits avec des organisations criminelles russes. L'entreprise à privatisée était ainsi, grâce à de multiples complicités, tombée dans le giron de la grande criminalité. Et le groupe européen WB put dire adieu à ses velléités de développement à court terme dans ce pays.

\section{Type 5: la chasse aux savoir-faire}

Cette chasse consiste à s'emparer des actifs intangibles de l'entreprise, qu'ils soient protégés ou non: brevets, marques, droits d'auteur ou savoir-faire propres à l'entreprise. Vieille comme le monde économique par le biais des activités d'espionnage industriel, elle est aujourd'hui contrôlée en grande partie par des organisations criminelles qui revendent l'actif dérobé ou l'utilisent pour développer leurs propres activités.
La contrefaçon, qui est devenue aujourd'hui indissociable de la contrebande et de la corruption, fait de plus en plus recours à des organisations criminelles (Pons, 2006) et entre ainsi dans cette catégorie.

\section{Type 6: la chasse au symbole}

La chasse à l'image correspond au cas où l'entreprise est perçue comme un symbole à détruire: symbole du capitalisme, symbole représentatif d'un pays comme les États-Unis, symbole du malheur pour les

6. L'attentat perpétré pour causer la mort d'un ou plusieurs employés sera classé dans la catégorie " chasse au symbole », car il est en général pratiqué par des organisations particulières pour suivant un objectif particulier: détruire. 


\section{KIDNAPPING EN COLOMBIE}

La scène se passe à Londres, au siège d'une compagnie d'assurances. Un émissaire de l'IRA (Irish Revolutionary Army) rencontre un expert en risque criminel employé chez l'assureur. L'émissaire fournit les preuves concernant le kidnapping d'un cadre expatrié d'une grande compagnie automobile Japonaise à Bogota, quatre semaines avant cette rencontre. La compagnie du cadre enlevé a assuré ses expatriés contre le kidnapping auprès de la compagnie d'assurances. L'émissaire explique que l'enlèvement a été perpétré par l'ELN (Ejército de Liberacion Nacional; Armée de libération nationale) et non pas par les FARC (Fuerzas Armadas Revolucionarias de Colombia; Forces armées révolutionnaires de Colombie). L'émissaire a apporté le portefeuille de l'expatrié ainsi qu'un film vidéo daté prouvant que l'otage est encore vivant et détenu par l'ELN. Il remet aussi un message manuscrit, estampillé ELN, exigeant une rançon de 800000 dollars contre libération de l'otage.

L'assureur contacte l'entreprise employeur de l'expatrié. Ils conviennent d'un plan d'action. L'assureur contacte aussi la société de sécurité spécialisée avec laquelle il a un partenariat. Cette société possède la compétence pour négocier avec l'organisation criminelle.

Pendant ce temps, le responsable des ressources humaines du fabricant automobile contacte la femme du cadre pour donner des nouvelles rassurantes: l'otage est a priori en vie. L'épouse sera tenue au courant de l'issue des négociations. Elle doit garder l'affaire secrète pour ne pas perturber leur déroulement.

Le cadre expatrié sera retrouvé dans un quartier riche de Bogota quatre semaines plus tard. L'assureur aura payé 600000 dollars contre sa libération.

populations d'une région, symbole du Nord face au Sud, etc. Les acteurs criminels agissant selon ce type ne sont pas des prédateurs qui cherchent à s'enrichir au détriment de l'entreprise, contrairement à ceux agissant selon les autres types. Ici les acteurs sont destructeurs et cherchent à anéantir tout ou partie de l'entreprise. Les terroristes entrent dans cette catégorie. Leur cible est soit un employé ou un groupe d'employés, soit des installations industrielles.

\section{Type 7: le parasitisme}

Le parasitisme consiste à utiliser la structure organisationnelle et les systèmes de gestion de l'entreprise pour développer des activités illégales. Dans cette catégorie entrent les opérations de blanchiment d'argent sale, le trafic d'êtres humains, le trafic de drogue, le trafic d'armes et le travail clandestin. L'acteur criminel déploie une logistique dans l'organisation de l'entreprise, à l'instar du ver solitaire qui se développe dans le corps humain. 


\section{CONTREFAÇON DE CIGARETTES CHEZ LES FUTURS ENTRANTS EUROPÉENS}

Fraîchement indépendant, le Monténégro est un acteur majeur du trafic de cigarettes en Europe. À telle enseigne que le véritable centre économique du pays n'est sans doute pas sa capitale Podgorica, mais le port de Bar, principal débouché du pays sur la mer Adriatique, à 1 heure 30 des côtes italiennes. Chaque jour entrent dans le port des cargos en provenance d'Amsterdam, Le Havre, Anvers, etc. Légalement importée au Monténégro, leur cargaison de cigarettes occidentales $T B$ est réacheminée par speed boats dès la nuit tombée vers la côte italienne des Pouilles. De là, elle est ensuite distribuée vers les marchés parallèles des grandes villes européennes par l'une des grandes mafias italiennes, la Sacra Corona Unita. Ce trafic porte sur plusieurs millions de cartouches de cigarettes, et génère un chiffre d'affaires criminel de plusieurs centaines de millions d'euros. Jusqu'à une période récente, ce trafic ne causait cependant pas de réel problème à $T B$ : archétypique d'un schéma de contrebande, il reposait en effet sur la vente de produits licites hors droits de douanes, et s'effectuait donc au préjudice de l'État et non du fabricant.

La situation est nettement différente depuis 2004, année où cette contrebande massive vers l'Europe occidentale s'est doublée d'une contrefaçon croissante des cigarettes $T B$ en Europe balkanique. Copiées dans les usines de tabac de Serbie, de Macédoine, de Croatie et de Bulgarie, des millions de cigarettes $T B$ sont avant tout vendues sur les marchés régionaux, créant d'importants obstacles au positionnement de $T B$ dans les Balkans. Mais des volumes croissants de $T B$ sont également exportés vers l'Europe occidentale via le « hub » Port de Bar - Sacra Corona Unita, où les cigarettes contrefaites concurrencent progressivement la marque sur son marché domestique.

La nature des acteurs du trafic et de la contrefaçon de ses produits complique considérablement les possibilités de réaction de $T B$ à cette prédation. Aux côtés des trafics de drogue, d'armes et d'êtres humains, la contrefaçon des cigarettes dans les Balkans est désormais une des « business units » d'organisations puissantes: crimes organisés serbe, croate, bulgare, et surtout mafia albanaise. Comme indiqué, le stake holder italien du hub est également une mafia. Son alter ego monténégrin étant, lui, constitué du plus proche entourage du chef d'un état devant prochainement entrer dans le processus d'adhésion à l'Union européenne... 


\section{TENTATIVES D'ATTENTAT CONTRE LE RALLYE PARIS-DAKAR}

Propriétaire de journaux tels que L'Équipe (premier tirage quotidien français) et Le Parisien - Aujourd'hui en France (premier quotidien d'actualité) le groupe Éditions Philippe Amaury est l'un des acteurs majeurs des médias en France. Par sa filiale ASO (Amaury Sport Organisation), le groupe Amaury est également leader du sport business en France, avec des événements sportifs tels que le Tour de France, l'Open de golf de France, le Marathon de Paris et le Rallye Paris-Dakar. Avec 5 millions de téléspectateurs chaque jour dans 187 pays, le rallye Paris-Dakar est une cible de choix pour une organisation terroriste voulant frapper un symbole occidental fort. Et en effet:

Janvier 2000 : le rallye Paris-Dakar quitte la capitale sénégalaise pour rallier Le Caire, selon un tracé encore inédit. Après quelques jours de course, les services secrets français et américains informent le ministère des Affaires étrangères que de forts risques d'attentat pèsent sur le rallye. Mokhtar Belmokhtar, alias Belouaer (« le borgne »), émir de la $6^{\mathrm{e}}$ région du $\mathrm{GSPC}^{7}$, a positionné plusieurs dizaines de ses hommes au sud de la frontière Algérie - Niger. Ce commando terroriste devrait tenter de frapper la caravane du rallye ou le bivouac durant l'une des quatre étapes prévues au Niger. La direction du rallye décide l'annulation immédiate de ces étapes, et organise l'opération « Reine de Sabha», pont aérien transportant véhicules et concurrents du Niger à la Lybie.

Janvier 2001 : le Front Polisario, mouvement rebelle du Sahara occidental menace de s'attaquer au rallye s'il traverse sa zone d'implantation, entre Maroc et Mauritanie.

Janvier 2004: Tarek Ibn Ziad, émir de la $5^{\mathrm{e}}$ région du GSPC planifie une attaque terroriste contre le rallye lors de son passage dans le nord du Mali. Sur les conseils du ministère français des Affaires étrangères, l'organisation du rallye décide d'annuler les deux étapes traversant le pays.

Novembre 2006: les services secrets français détectent une possible préparation d'attaque terroriste du rallye par le GSPC à l'ouest du Mali.

La rentabilité de l'événement est assurée par les droits de retransmission télévisée des plus de 550 heures d'images tournées lors du rallye. Après deux étapes annulées, celle-ci n'est plus garantie, des pénalités pouvant même être contractuellement exigées par les chaînes de télévision. Au-delà de coûts directs importants (une opération du type «Reine de Sabha » coute entre 4 et 5 millions d'euros, le risque terroriste pesant constamment sur le rallye remet donc en question le modèle économique même de ce type de grand événement.

7. GSPC: Groupement salafiste pour la prédication et le combat. Dernière organisation terroriste algérienne depuis le démantèlement des Groupes islamiques armés (GIA) par les forces de sécurité algériennes, le GSPC reste particulièrement actif en Algérie et en Europe. Affilié à Al-Qaida, le GSPC a proféré des menaces terroristes à plusieurs reprises contre les intérêts occidentaux présents dans sa zone d'action, les dernières d'entre elles datant du mois de novembre 2006. 


\section{-TRAFIC DE DROGUE CHEZ UN PRODUCTEUR DE MÉTAUX}

Tout commença avec la mort d'un plongeur sous-marin en Jamaïque. MET, fabriquant de métaux, possédait un gisement de matière première dans ce pays. Les matières étaient extraites du sol, chargées sur des tankers appartenant à l'entreprise, qui naviguaient jusqu'en Amérique du Nord ou les matières étaient transformées en métal. À chaque chargement, deux types de contrôle étaient exercés. Les douanes contrôlaient la cargaison du navire et faisaient une inspection totale des soutes avant appareillage. Une équipe de plongeurs sousmarins, employés par MET, faisaient, eux, une inspection extérieure de la coque du bateau. L'enquête sur la mort du plongeur montra qu'il était en relation avec un gang de Kingston impliqué dans le trafic de drogue effectué à partir de la Jamaïque. Ces criminels faisaient apparemment pression sur lui pour qu'il agisse selon leurs desiderata. Une inspection des navires contrôlés habituellement par ce plongeur fut alors diligentée. Les autorités constatèrent qu'un conteneur étanche avait été soudé sous la coque d'un bateau. L'histoire fut alors reconstituée.

Le plongeur devait placer la drogue dans le conteneur, sur ordre des criminels. Dans le rapport d'inspection interne à MET, il signalait que tout était normal. La drogue voyageait donc avec le minerai de l'entreprise jusqu'en Amérique du Nord. Là-bas, d'autres plongeurs à la solde du gang jamaïquain récupéraient la cargaison illicite. Le plongeur de MET avait été abattu pour l'exemple, parce qu'il refusait de continuer à coopérer avec les criminels.

En synthèse, cette typologie représente une tentative d'ordonnancement des crimes perpétrés par les groupes criminels organisés. Nous avons essayé autant que possible d'être exhaustifs. Pour un seul type, la chasse au symbole, les criminels cherchent à détruire l'entreprise. Pour les autres types, ils agissent en prédateurs, ponctionnant les ressources visées au sein de leur proie ou se servant de son organisation. Ces prédateurs ont donc tout intérêt à maintenir en vie cette proie qui peut leur apporter ainsi des revenus (ou autre objectif) récurrents. Le cas du pizzo, système d'extorsion simple géré par les mafias italiennes, illustre notre propos. Le pizzo (la pièce) est appliqué à tout commerce ou entreprise présente sur le territoire de la mafia. Il est calculé pour ne pas créer de difficultés à l'entreprise rackettée.
Il est versé par celle-ci avec une périodicité fixée par le groupe criminel.

Autre considération: il peut donc y avoir combinaison de types pour une entreprise sur un territoire donné, comme les compagnies pétrolières dans le delta du Niger qui font face aux communautés locales (chasse au trésor) et aux armées rebelles dégénérées (chasse au produit).

Enfin, l'éventail des types montre que l'entreprise peut être attaquée de toutes parts: marchés, produits, organisation, ressources financières, humaines, techniques ou technologiques. Elle est donc a priori vulnérable de multiples façons.

\section{Impacts de l'action criminelle}

La nature des impacts micro-économiques a été analysée grâce aux études de cas. L'ana- 
lyse comparée de ces cas montre que la nature des impacts diffèrent selon les types de crime commis. Cette réflexion nous invite à formuler la proposition ci-dessous.

P1. La nature des impacts provoqués par l'action criminelle sur l'entreprise dépend du type d'action mis en œuvre par le groupe criminel organisé.

Les impacts sont divers. Nous nous sommes appuyés sur des modèles utilisés en management stratégique pour les classer. Ainsi nous avons retenu les six domaines d'impact suivants: stratégie, ressources humaines (et en particulier comportement des employés), systèmes de gestion, responsabilité légale (civile ou pénale) du dirigeant ou de l'organisation, et enfin deux indicateurs de performance: performance boursière et résultat net.

Les propositions détaillées concernant la nature des impacts par type d'action sont résumées dans la figure 1.
Les propositions détaillées liant un type de crime à ses impacts probables sont détaillées ci-dessous.

P1.1. L'impact micro-économique de la chasse au trésor consiste en une baisse $d u$ résultat net.

L'extorsion se traduit par une perte dans le compte de résultat. Au-delà, elle peut poser des problèmes de financement de la trésorerie immédiate, et requérir le recours à davantage d'emprunts bancaires, dont il faudra payer les intérêts. Tel est le principal impact identifié.

P1.2. L'impact micro-économique de la chasse au produit consiste en une baisse $d u$ résultat net.

Le vol et la piraterie sont des risques qui, en règle générale, sont transférables à un assureur. De fait, l'impact majeur consiste en une hausse des coûts d'assurance qui accroît donc les charges supportées par

Figure 1 - Nature des impacts par type d'action criminelle

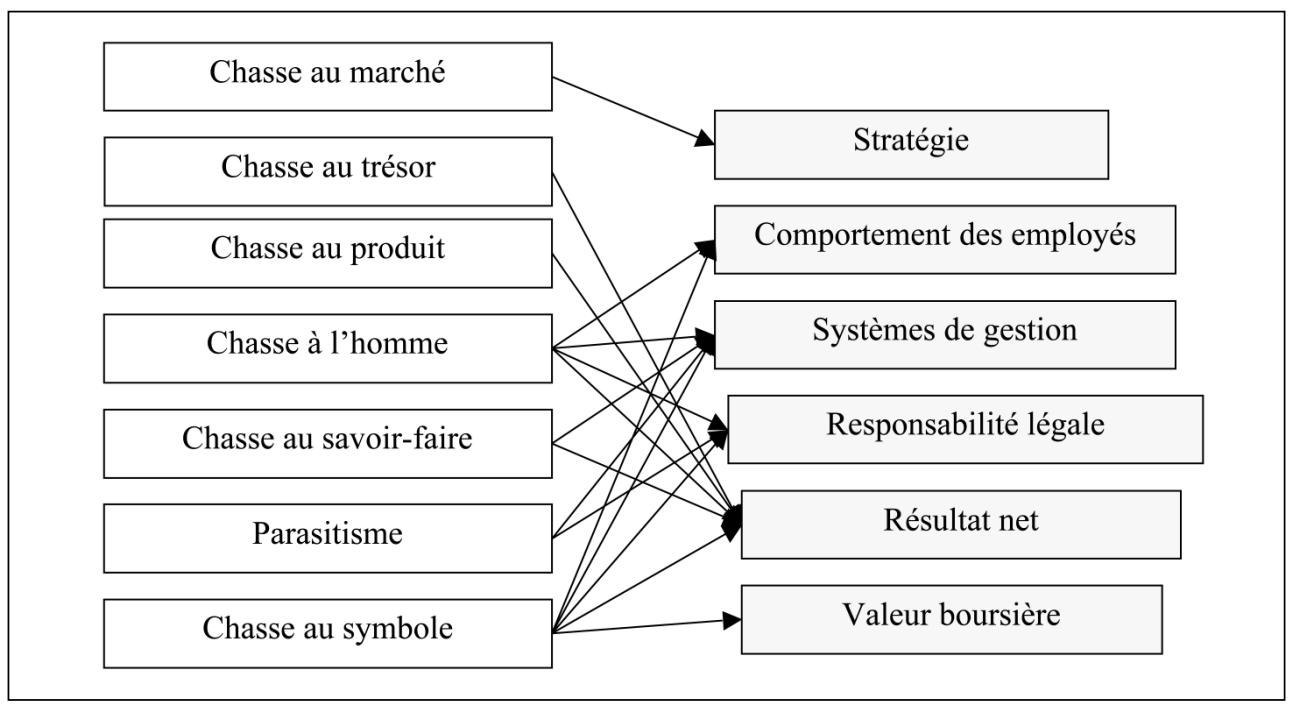


l'entreprise et comptabilisées dans le compte de résultat.

\section{P1.3. L'impact micro-économique de la} chasse au marché consiste en une révision de la stratégie

Le piratage des marchés et privatisations contraint l'entreprise à trouver de nouveaux axes de développement pour l'entreprise. L'impossibilité de conquérir un marché n'influence pas la performance actuelle: c'est un surcroît de performance économique espérée qui échappe à l'entreprise.

P1.4. L'impact micro-économique de la chasse à l'homme consiste en:

- une baisse du résultat net,

- un accroissement du risque juridique,

- un changement de comportement des employés,

- une révision des systèmes de gestion.

Le paiement d'une rançon affecte les comptes de l'entreprise. Si elle a transféré le risque auprès d'un assureur, ce sont les primes d'assurance qui devraient augmenter suite à des enlèvements. Les préjudices moraux, et parfois physiques, subis par l'otage peuvent engager la responsabilité civile de l'entreprise et/ou la responsabilité pénale des dirigeants: en assimilant le kidnapping à un accident du travail, l'employeur peut être reconnu coupable de faute inexcusable parce qu'il n'avait pas pris toutes les mesures nécessaires pour assurer la sécurité de ses employés (Collard, 2005). Les enlèvements ont aussi pour effet de créer peurs et tensions chez les autres employés de l'entreprise. Ils créent aussi des difficultés pour recruter et envoyés des cadres sur le territoire du crime. Ces réactions ont une autre conséquence: l'augmentation des primes d'expatriation, et donc des charges de personnel, qui influencent aussi le résultat net. Enfin, l'entreprise confrontée à des enlèvements est en générale contrainte à modifier certains systèmes de gestion: gestion des ressources humaines, systèmes de sécurité.

P1.5. L'impact micro-économique de la chasse au savoir-faire consiste en une baisse du résultat net et une révision des systèmes de gestion

La perte d'une source d'avantage concurrentiel a des répercussions négatives sur la performance économique, que le savoir soit utilisé par des concurrents ou utilisé pour développer des activités de contrefaçon. $\mathrm{Ne}$ possédant plus d'exclusivité, l'offre de l'entreprise perd son attrait auprès des clients. La conséquence est une chute probable du chiffre d'affaires et, toutes choses (charges) égales par ailleurs, une diminution du résultat net. La perte d'un tel avantage entraîne l'entreprise à augmenter les moyens de protection de ses innovations et sources de différenciation; la gestion de l'information devient plus rigoureuse, les protections légales de la propriété sont utilisées, des clauses d'exclusivité sont inscrites dans les contrats de travail. Certains systèmes de gestion sont donc modifiés.

P1.6. L'impact micro-économique de la chasse au symbole consiste en:

- une baisse du résultat net,

- une baisse de la valeur de l'entreprise,

- une révision des systèmes de gestion,

- un changement de comportement des employés,

- un accroissement du risque juridique.

La destruction de sites ou la mort d'employés augmente les coûts d'assurance et peut, à l'instar de la chasse à l'homme, augmenter les primes de risque pour les employés représentant des cibles potentielles. Ces crimes peuvent aussi paralyser la gestion opérationnelle de l'entreprise et 
créer ainsi un manque à gagner important. De fait, puisque de tels attentats sont médiatisés, c'est la valeur boursière de l'entreprise cotée qui devrait immédiatement baisser. Le moral des employés est atteint et les affectations dans des implantations jugées dangereuses deviennent difficiles à mettre en œuvre. Les attentats entraînent aussi une modification des systèmes de gestion: renforcement de la sécurité, modification de la politique de gestion des ressources humaines. Enfin, en particulier lorsque des employés sont décédés, la responsabilité civile de l'entreprise et la responsabilité pénale des dirigeants peuvent être engagées (Collard, 2005).

\section{P1.7. L'impact micro-économique du para-} sitisme consiste en :

- une révision des systèmes de gestion de l'entreprise,

- un accroissement du risque juridique.

L'objectif des parasites n'est pas de partager les bénéfices de l'entreprise. Il s'agit d'utiliser son organisation pour développer des activités ayant leur propre rentabilité. Le crime n'a donc pas d'impact majeur sur la performance économique. En revanche, la découverte d'agissements parasites contraint l'entreprise à modifier certains systèmes de gestion. Par exemple la modification des trajets et des comportements des conducteurs dans une entreprise de logistique routière. Par exemple, un changement de la politique de recrutement et de gestion des employés « exposés », ceux dont la complicité pourrait être recherchée par les criminels. Enfin, le risque juridique est important: en France, le détenteur des marchandises de fraude est réputé responsable de la fraude (Bauer, 2005, citant l'article 392 du code des douanes).
Si ces propositions étaient corroborées, cela signifierait qu'il n'y a pas une seule solution envisageable face au crime. La réponse, aussi bien préventive que curative, devrait être adaptée selon la nature des impacts prévisibles. Par exemple, une action criminelle comme la chasse à l'homme induit un risque d'engagement de la responsabilité légale du dirigeant, et requiert donc de mettre en place toutes les mesures anticipatrices nécessaires pour à la fois prévenir la menace et éviter la mise en cause du dirigeant.

\section{Résultat complémentaire : faire face simultanément à divers types de crime}

La spécificité de chaque type d'action criminelle induit donc que l'entreprise peut faire face à une grande complexité lorsqu'elle affronte simultanément plusieurs types d'actions. Tel est le cas de certaines industries comme les secteurs pétroliers ou para-pétroliers où les firmes font face, au minimum, à la chasse au trésor, la chasse à l'homme, la chasse au produit et la chasse au marché. L'action criminelle sur les organisations de ces secteurs a donc a priori des conséquences à la fois sur leur performance, leur management stratégique et leur responsabilité juridique. Ces impacts ne sont pas tous localisés, puisque certains types d'action ne sont pas associables à un territoire particulier de la planète (voir le tableau 1). De fait, ce sont la compétitivité locale et la compétitivité globale de l'entreprise qui peuvent être menacées. C'est pourquoi la menace criminelle gagnerait à être intégrée dans la réflexion stratégique pour de telles organisations. Ce constat nous incite à formuler une proposition complémentaire : 
P2. Plus le nombre de types d'action criminelle rencontrés simultanément est important, plus la performance économique de l'entreprise est liée à la prise en compte du risque criminel dans la réflexion stratégique.

Si cette hypothèse était corroborée, le risque criminel pourrait dorénavant être considéré comme un risque systémique, appartenant à l'univers dans lequel se meut l'entreprise.

\section{Discussion et axes de recherche}

Notre modèle théorique de l'action criminelle sur l'entreprise tient en trois points :

- il existe une diversité dans l'action criminelle, qui peut être réduite à sept types d'action;

- la nature des impacts du crime sur les organisations dépend du type d'action criminelle mis en œuvre;

- la performance économique des entreprises soumises simultanément à plusieurs types d'action criminelle dépend de la prise en compte du risque criminel dans la réflexion stratégique globale.

Notre modèle suggère que le terrorisme ne peut être assimilé à la menace criminelle. Depuis le 11 septembre, les regards et les efforts se sont déployés dans les pays industrialisés pour faire face à cette menace. Mais en définitive, c'est simplement un type d'action parmi de nombreux autres. Faire l'amalgame est dangereux, car la nature des impacts sur l'entreprise dépend du type d'action criminelle. En criminologie, on parlerait d'une balistique différente selon l'action. Si la balistique est différente, la protection contre la menace requiert des solutions différentes. On n'évite pas une asphyxie au gaz comme on évite un projectile de mortier.
En complément, l'exposition simultanée de certaines industries à de multiples actions criminelles suggère une intégration de la menace criminelle dans la stratégie de l'entreprise. Cette intégration serait nécessaire pour coordonner la mise en place de solutions adaptées à chaque problème, qu'elles soient appliquées au niveau local (à une filiale) ou global (à toutes les entités composant l'entreprise), ou aux deux à la fois. Développer une simple stratégie contre le terrorisme risque alors de laisser libre cours à d'autres types d'action criminelle contre lesquels aucun dispositif n'a été conçu.

D'autre part, nos résultats soulignent que l'action criminelle sur l'entreprise entraîne de nombreux dégâts collatéraux. Attendu que :

- les organisations criminelles s'attaquent toujours au maillon faible, tout employé est cible potentielle d'intimidation ou de pression afin de travailler pour l'organisation criminelle, même si certains sont plus exposés que d'autres: expatriés dans pays à risque, traders à New York ou Londres ;

- l'action criminelle sur l'entreprise tend à diminuer ses profits et donc, soit à réduire les budgets consacrés aux augmentations de rémunération et autres avantages sociaux, soit à réduire les coûts et éventuellement licencier une partie de ses effectifs;

- les grandes organisations criminelles gèrent des sociétés d'apparence légale qui viennent concurrencer les « vraies » entreprises et donc les affaiblir.

Chacun en tant qu'employé, peut subir les dommages de l'attaque criminelle sur son entreprise, au niveau de sa probité et de son éthique, au niveau de son porte-monnaie, voire au niveau de son emploi. 
Attendu que :

- le coût des mesures de protection mises en œuvre par les entreprises contre le crime, peut se répercuter à terme par une augmentation des prix;

- les modes d'action visant l'entreprise peuvent toucher les clients - empoisonnement alimentaire, faux médicaments fabriqués clandestinement, incapacité à délivrer les produits, etc.

Chacun, en tant que consommateur, peut subir les dommages de l'attaque criminelle sur les entreprises, au niveau de son portemonnaie, de sa santé, voire de sa survie.

Attendu que certains criminels contrefacteurs produisent aujourd'hui des composants automobiles et destinés aux avions; chacun, en tant que touriste, peut subir les dommages de l'action criminelle sur l'entreprise au niveau de sa survie.

L'action criminelle sur les organisations menace donc, par ricochet, les différentes facettes de la vie des individus. Dès lors la lutte contre le crime constitue un devoir des entreprises. À l'heure où le monde des affaires met en avant la responsabilité sociale de l'entreprise, il serait pertinent d'inclure dans cette démarche un volet consacré à la lutte contre le crime et ses répercussions dans nos sociétés.

Toutefois, cette lutte contre le crime pose la question de la légitimité des pratiques: pratiques des organisations « honnêtes » et pratiques des groupes criminels. Les communautés du delta du Niger considèrent la chasse au trésor comme une pratique légitime: compte tenu des bouleversements écologiques - pollution des zones de pêche par exemple - et économiques - enrichissement des politiques locaux corrompus au détriment des populations - provoqués par le développement du secteur pétrolier, il est
« normal » que les communautés locales obtiennent leur part du gâteau. Faut-il alors parler de crime commis par ces communautés? Faut-il parler de compensation légitime? Selon les patrons des organisations multinationales que nous avons interrogés, les réflexions se tournent aujourd'hui vers le développement durable: comment vivre tous ensemble, en paix et en bonne intelligence, dans la région du delta. Il y a donc interpénétration forte de deux mondes dits parallèles: celui qualifié de l'action légale et celui qualifié de l'action illégale ou criminelle.

La frontière est donc tenue entre légitimité et illégitimité de l'action, entre légalité et illégalité. À l'instar de l'exemple cité cidessus, les solutions à inventer reposent sur une compréhension préalable des motivations génératrices de l'action criminelle. Une communauté locale qui pratique la chasse au produit ou au trésor n'a pas les mêmes motivations qu'une mafia ou un cartel qui pratique le parasitisme. Le concept de crime, défini initialement de manière universaliste dans ce papier, peut aussi être envisagé sous un angle plus contingent.

Ces discussions et développements émanent d'une recherche dont il convient de souligner quelques limites :

- le nombre de cas étudiés est faible relativement au nombre de catégories dans notre typologie: 10 pour 7 . Bien que nous ayons utilisé la revue de presse pour tenter de valider notre découpage en types, la capacité à reproduire l'analyse pour chaque type, jusqu'à saturation de la théorie, aurait fourni une validation plus solide de notre modèle; soulignons toutefois la difficulté à trouver des cas, car peu d'entreprises souhaitent évoquer les sujets criminels; 
- si la nature des impacts est importante, l'ampleur de deux-ci l'est tout autant. En effet, il est possible que certaines actions criminelles aient un impact bien plus négligeable que d'autres. Nous n'avons pas mesuré la taille des impacts dans cette recherche. De fait, le modèle qui présente de façon identique chaque type donne peutêtre une fausse représentation de la réalité, en matière d'importance relative de chaque risque criminel.

Ces limitations devraient être prises en compte dans les recherches futures. Plusieurs voies sont possibles. Nous en suggérons deux.

Tout d'abord, il est possible de lancer une réplication par étude de cas. Dans chaque catégorie de notre typologie, deux cas seraient sélectionnés et analysés dans le but d'étudier la nature et l'ampleur des impacts du crime étudié. La mesure des impacts pourrait être effectuée. Ce projet serait donc à la fois une réplication de notre travail empirique, avec un enrichissement possible du cadre d'analyse.

Ensuite, une recherche de type quantitative est envisageable, pour tester le modèle sur un vaste échantillon d'organisations. Les variables citées sont toutes facilement mesurables, à travers des données factuelles ou en recueillant les perceptions des dirigeants (par exemple sur la révision de la stratégie ou la révision des systèmes de gestion). La principale difficulté qui risque d'être rencontrée réside dans le taux de réponse. Il faudra réussir à convaincre les dirigeants à fournir des informations sur les activités criminelles auxquelles ils ont été confrontés.

\section{CONCLUSION}

Nos investigations montrent que l'entreprise peut être attaquée de tout côté par des groupes criminels organisés, qu'ils poursuivent un but idéologique, veulent s'enrichir ou s'appuyer sur une entité honnête pour développer leurs pratiques frauduleuses. Face à la créativité de ces groupes criminels, les organisations apparaissent aujourd'hui des proies vulnérables: on peut s'emparer de leurs ressources humaines, financières, technologiques; on peut détruire leurs sites ou tuer leurs hommes; on peut détourner leur logistique pour pratiquer des trafics prohibés.

La gestion de ces risques criminels est requise, probablement au plus haut niveau de l'organisation dans des industries particulièrement menacées. Dans de tels secteurs, la capacité à gérer le crime a toutes chances d'influencer la performance économique. Si elle ne constitue pas une source d'avantage concurrentiel à proprement parler, cette capacité permet au moins d'éviter la création d'un désavantage. Car cette recherche suggère que le monde des affaires doit apprendre désormais à vivre avec la sphère dite criminelle et non plus en parallèle. 


\section{BiBLIOGRAPHIE}

Bauer A., «Sécurité, crime et entreprises: le nouveau chaos mondial », Défense Nationale et Sécurité Collective, $\mathrm{n}^{\circ}$ 3, supplément spécial, 2005, p. 9-16.

Claveau N., Séville M., « Le tireur de sonnette d'alarme, un acteur-clé du management stratégique? », XIII Conférence de l'Association Internationale de Management Stratégique, Normandie, Vallée de Seine, juin 2004.

Collard C., « Terrorisme et engagement de la responsabilité de l'entreprise et de ses dirigeants », Défense Nationale et Sécurité Collective, n 3, supplément spécial, 2005, p. 33-42.

Czinkota M.R., Knight G.A., Liesch P.W., Steen J., "Positioning Terrorism in Management and Marketing: Research Propositions", Journal of International Management, vol. 11, $\mathrm{n}^{\circ}$ 4, 2005, p. 581-601.

Dacin T., DeSoucey M., Pozner J-E., "Profits to prophets: feeding ideologies at the fringe", Conférence de Academy of Management, Symposium "Code makers and code breakers", Philadelphia, août 2007.

Deakin S., Konzelman S. J., "Learning from Enron", Corporate Governance: an International Review, vol. 12, $\mathrm{n}^{\circ} 2$, 2004, p. 134-142.

Doh J.P., Rodriguez P., Uhlenbruck K., Collins J., Eden L., "Coping with corruption in foreign markets", Academy of Management Executive, vol. 17, n 1, 2003, p. 114-127.

Eisenhardt K. M., "Building theories from case study research", Academy of Management Review, vol. 14, n 4, 1989, p. 532-550.

Gundlash M.J., Douglas S.C., Martinko M.J., "The decision to blow the whistle: a social information processing framework", Academy of Management Review, vol. 28, n 1, 2003, p. 107-123.

Kaufman A., Englander E., "A team production model of corporate governance", Academy of Management Executive, vol. 19, n 3, 2005, p. 9-22.

Morrison J., "Legislating for good corporate governance", Journal of Corporate Citizenship, 15, 2004, p. 121-133.

Pierrat J., Sargos A., Yakusa, Flammarion, Paris, 2005.

Pons N., Cols blancs et mains sales - Économie criminelle, mode d'emploi, Odile Jacob, Paris, 2006.

Raufer X., La Camorra, La Table Ronde, Paris, 2005.

Raufer X., Quéré S., Le crime organisé, Presses Universitaires de France, Paris, 2005.

Schleifer A., Vishny R.W., “Corruption”, Quarterly Journal of Economics, août 1993, p. 599-617.

Spich R., Grosse R., "How homeland security affect U.S. firms' international competitiveness?", Journal of International Management, 11, 2005, p. 457-476.

Uhlenbruck K., Rodriguez P., Doh J., Eden L., "The Impact of corruption on entry strategy: evidence from telecommunication projects in emerging economies", Organization Science, vol. $17 \mathrm{n}^{\circ} 3,2006$, p. 402-414.

Vershoor C., "Are whistleblowers heroes or just doing their jobs?", Strategic Finance, vol. 84, n 9, 2003, p. 18-19. 